\title{
Micromachine Wedge Stepping Motor
}

\author{
James J. Allen and Heather K. Schriner \\ Sandia National Laboratories \\ Intelligent Micromachine Department \\ Albuquerque, NM 87185-1080 \\ http://www.mdl.sandia.gov/Micromachine
}

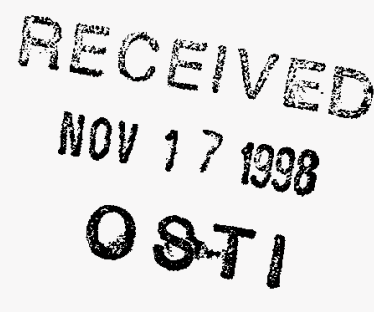

\section{Abstract}

A wedge stepping motor, which will index a mechanism, has been designed and fabricated in the surface micromachine SUMMiT. (Sandia Ultra-planar Multi-level MEMS Technology) [1] process. This device has demonstrated the ability to index one gear tooth at a time with speeds up to 205 teeth/sec. The wedge stepper motor has the following features, which will be useful in a number of applications.

- The ability to precisely position mechanical components.

- Simple pulse signals can be used for operation.

- Only 2 drive signals are required for operation.

- Torque and precision capabilities increase with device size

- The device to be indexed is restrained at all times by the wedge shaped tooth that is used for actuation.

This paper will discuss the theory of operation and design of the wedge stepping motor. The fabrication and testing of the device will also be presented.

\section{Introduction}

An indexing mechanism is a fundamental device that can be incorporated into many systems to provide dwell in the device motion, for example, indexing a numbered rotor in an odometer or counter. The indexing function can be achieved by a number of mechanisms such as a cam, geneva ratchet, mutilated pinion or an escapement mechanism [2]. There are many possible approaches available to achieve this function; however, each approach has advantages or disadvantages such as speed, dynamic forces, smoothness of operation or wear. The ability to fabricate the device in a surface micromachine process is important for applications to be considered in this paper.

The electrostatic comb drive has become a fundamental actuator available for producing mechanical motion for surface micromachined devices. The microengine, which consists of two comb drive actuators and appropriate linkages, is capable of producing rotary motion and driving a number of devices such as gear trains, pop up mirrors, and shutters [3]. However, the microengine has limited capability for precision positioning at any location other than the fabricated position because the position is a function of load, friction and other variables as well as the applied voltage.

An application of particular interest to Sandia National Laboratories is the countermeshing gear discriminator [4]. This is a locking device based on the proper sequence of counter rotation of meshing gears (i.e. code) required to rotate a specified amount. The gears have three levels of teeth which are selectively present that specify the proper rotation sequence to unlock the device. This device depends on the proper positioning of the counter meshing gears for operation.

The wedge stepper motor was designed to provide precision indexing of mechanical components such as gear teeth. This paper will discuss the theory of operation and design of the wedge stepping motor. The fabrication and testing of the device will also be presented.

\section{Wedge Stepping Motor}

The wedge stepper motor shown in Figure 1 consists of an outer ring and a reciprocating 


\section{DISCLAIMER}

This report was prepared as an account of work sponsored by an agency of the United States Government. Neither the United States Government nor any agency thereof, nor any of their employees, make any warranty, express or implied, or assumes any legal liability or responsibility for the accuracy, completeness, or usefulness of any information, apparatus, product, or process disclosed, or represents that its use would not infringe privately owned rights. Reference herein to any specific commercial product, process, or service by trade name, trademark, manufacturer, or otherwise does not necessarily constitute or imply its endorsement, recommendation, or favoring by the United States Government or any agency thereof. The views and opinions of authors expressed herein do not necessarily state or reflect those of the United States Government or any agency thereof. 


\section{DISCLAIMER}

Portions of this document may be illegible in electronic image products. Images are produced from the best available original document. 
shuttle. The outer ring has internal teeth that are engaged by a tooth on each end of the reciprocating shuttle. The outer ring can have gear teeth on the outside or other means of engaging another mechanical member. Figure 2 shows a rotary version of the indexing stepper motor. However, a linear version of this concept may be realized as well.

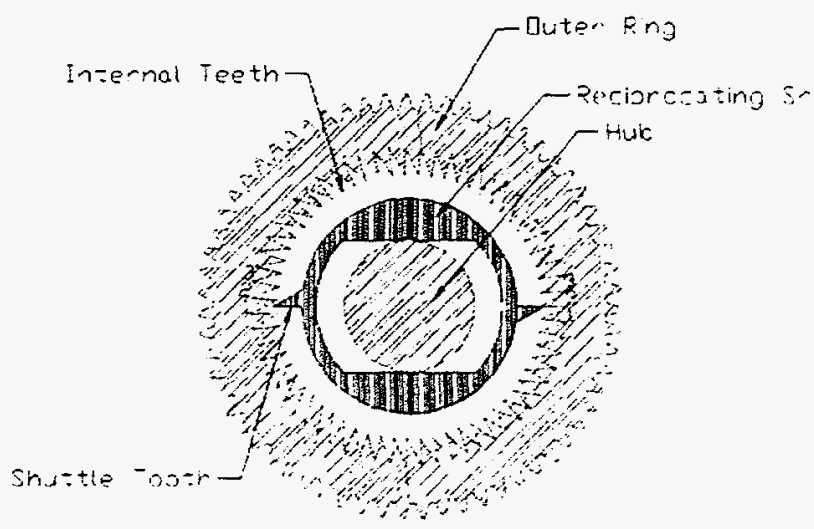

Figure 1. Schematic of the Wedge Stepping Motor.

This device can be used to index mechanical elements such as a rotary gear or a linear rack. The device actuation element is the reciprocating shuttle tooth, which engages internal teeth on the outer ring that will rotate the outer ring. The device can be designed such that the shuttle tooth is always at least partially inserted into the internal teeth, which will constrain the rotary position of the outer ring to prevent free rotation. The outer ring is most accurately positioned when the shuttle is at the extreme of its stroke. This indexing device will produce rotary motion in one direcrion. An externally actuated device as shown in Figure 2 may actuate the shuttle; however, the system could be designed to place the actuator inside the outer ring. This device is amenable to fabrication in a surface micromachine process, and it can be incorporated into desicns which require precise and repeatable positioning of micromechanical components.

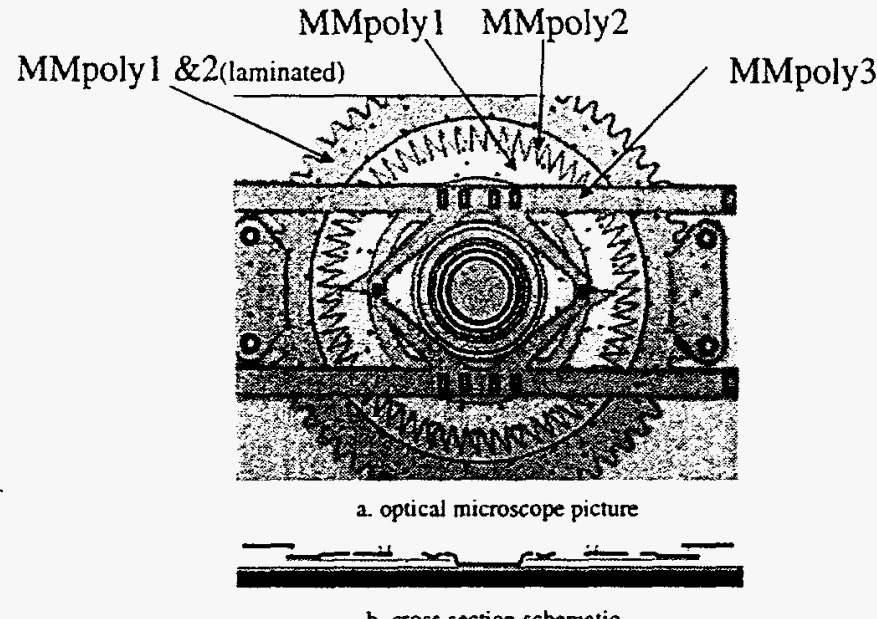

Figure 2. Wedge Stepper Motor

\section{Mechanical Design Issues}

The reciprocating tooth must interact with an odd number of internal teeth $(2 * \mathrm{~N}+1)$ for proper operation. The angles shown in Figure 4 show the geometry of the reciprocating tooth and the internal teeth. The angles are defined as follows

- $\theta_{L}$ - Angle spanned by the internal tooth land (optional internal tooth feature)

- $\theta_{\mathrm{G}}$ - Angle spanned by the internal tooth and the reciprocating tooth clearance, which is required for fabrication in the surface micro-machining process .

(i.e. $\theta_{\mathrm{G}} \geq 2 \cdot$ (Fabrication line space limit)

- $\quad \theta_{1}$ - Angle spanned by the inserted portion of the reciprocating tooth into the internal tooth.

- $\theta_{\mathrm{S}}$ - Angle spanned by the rotary motion produced by one stroke of the reciprocating tooth.

- $\lambda$ - Angle spanned by one internal tooth.

The relationship of the angle spanned by one internal tooth $(\lambda)$ to the other tooth angles $\left(\theta_{\mathrm{L}}, \theta_{\mathrm{G}}, \theta_{\mathrm{l}}, \theta_{\mathrm{S}}\right)$ is shown in equation 1 .

$$
\lambda=\theta_{\mathrm{L}}+\theta_{\mathrm{G}}+\theta_{\mathrm{I}}+\theta_{\mathrm{S}}
$$

To close the reciprocating tooth - internal tooth gap, and extract the reciprocating tooth from the opposite side, Figure $3 \mathrm{~b}$, the following relation shown in equation 2 must be satisfied.

$$
\theta_{\mathrm{G}} \geq \theta_{\mathrm{I}}
$$


Following a stroke, in order for the reciprocating tooth to be inserted in the opposite side of the internal gear, Figure $3 \mathrm{c}$, the following relation must be satisfied by the design.

$$
\theta_{S} \geq \theta_{L}+\theta_{1}
$$

A latch or use of a motion profile for the reciprocating tooth may be required for assured insertion of the reciprocating tooth on the reversing stroke during high-speed operation.

The mechanical advantage of the shuttle input force $\left(F_{i n}\right)$ to the transverse force $\left(F_{L}\right)$ on the outer ring is a function of the wedge angle $(\alpha)$ and the coefficient of friction $(\mu)$ between the interacting parts. The relationship, equation 4 , between these variables can be obtained by an elementary static analysis of the cevice. The minimum practical wedge angle achievable is constrained by the line space limitalions of the fabrication process. Figure 5 show the dependence of the mechanical advantage on $\alpha$ and $\mu$. To obtain a mechanical advantage of approximately 1 with a coefficient of friction $(\mu)$ of $0.3-0.5$, a value of 27 degrees was chosen for $\alpha$.

$$
\frac{F_{L}}{F_{\text {in }}}=\frac{\cos \alpha}{\sin \alpha+\mu \cos \alpha}-\frac{\mu \cos \alpha}{\sin \alpha+\mu \cos \alpha}
$$

The wedge stepper motor is capable of accurately positioning a mechanical element such as a gear or rack in a known repeatable position. This indexing device can be fabricated in surface micromachine processes, and can be driven by actuators, which have been developed in the surface micromachine technology. The fabrication of the wedge stepping motor shown in Figure 2 requires the use of three independent mechanical layers and a ground plane.

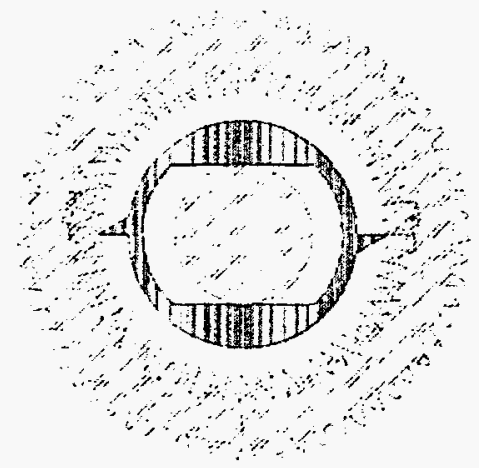

a. Starting Position

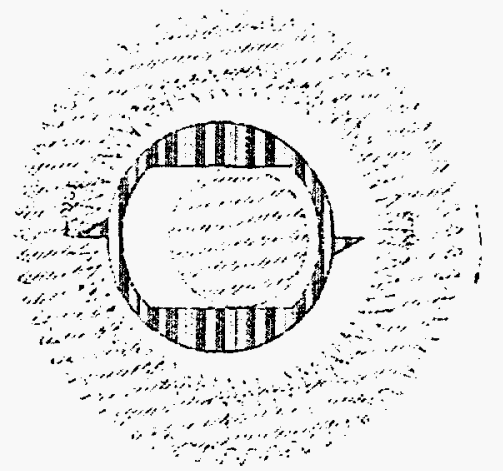

b. Left Stroke

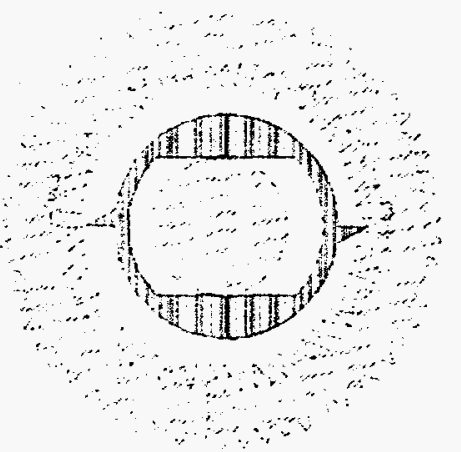

c. Right Stroke

Figure 3. Operation Sequence for the Wedge Stepper Motor

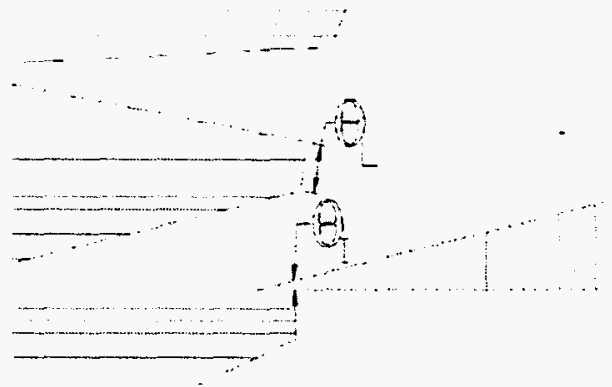

a. Left Tooth

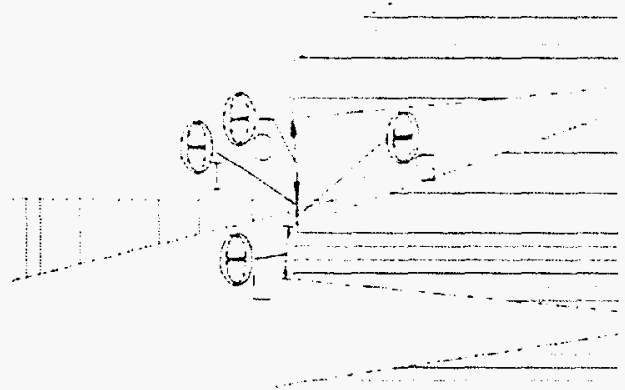

b. Right Tooth

Figure 4. Internal Tooth Geometry 


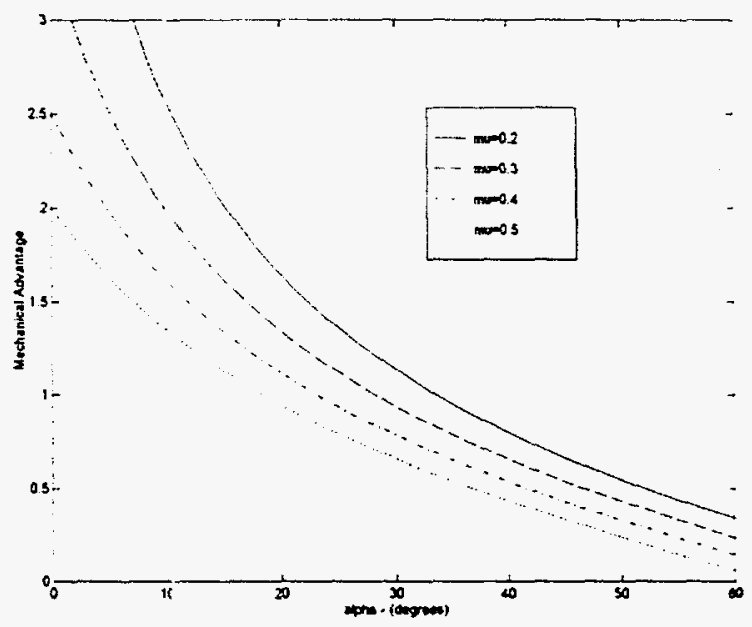

Figure 5. Mechanical Advantage versus Wedge Angle $(\alpha)$ and Coefficient of Friction $(\mu)$.

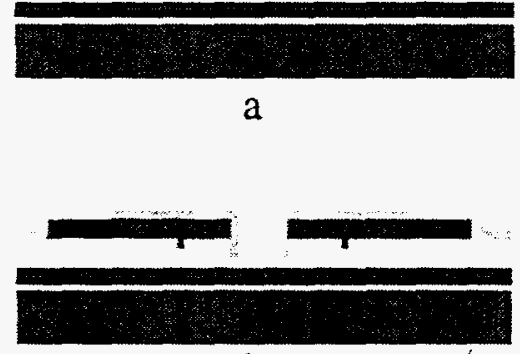

b

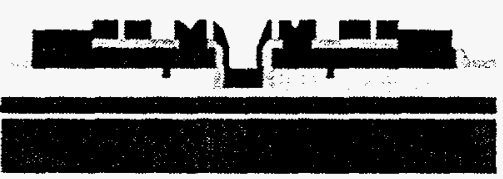

C

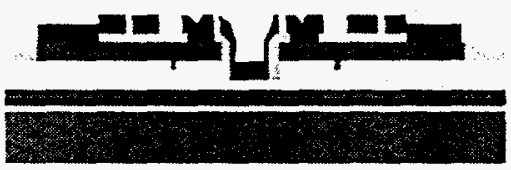

d

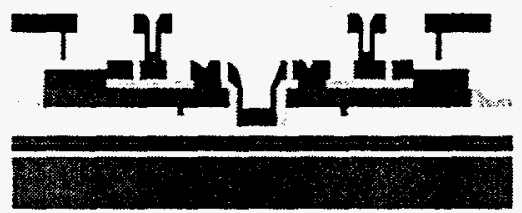

e

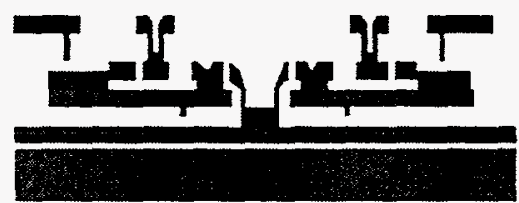

f

Figure 6. SUMMiT Process Fabrication Sequence.

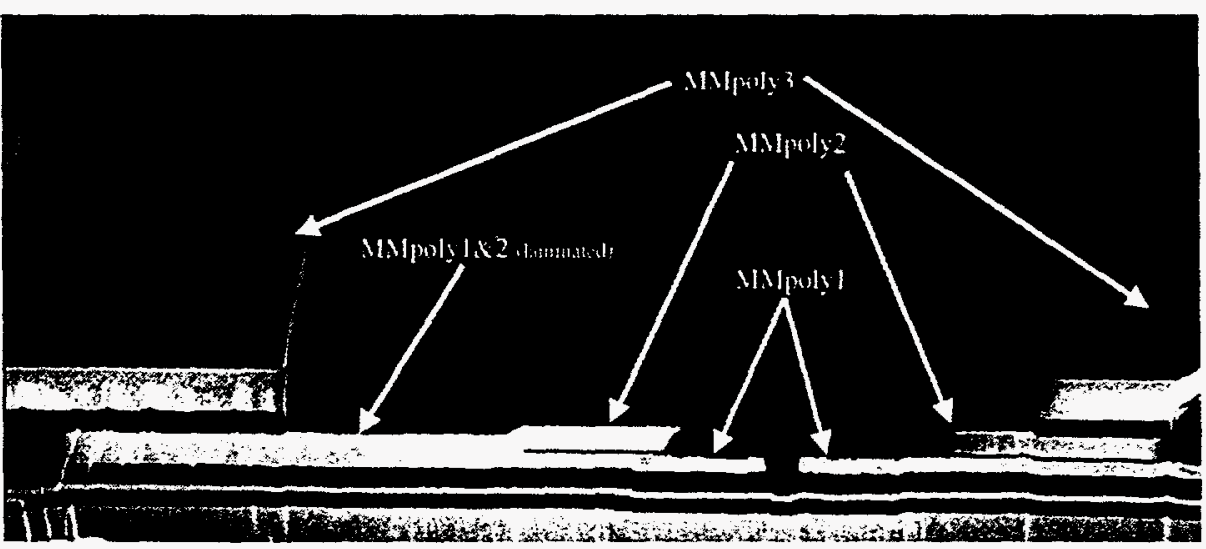

Figure 7. Focus Ion Beam Cross Section of the Wedge Stepping Motor 


\section{SUMMiT Fabrication Processi}

The SUMMiT surface micromachine fabrication process, which has the ability to fabricate the wedge stepping motor, will now be described. The SUMMiT fabrication process begins with a bare n-type, $<100\rangle$ silicon wafer. A $0.63 \mu \mathrm{m}$ layer of silicon dioxide $\left(\mathrm{SiO}_{2}\right)$ is thermally grown on the bare wafer. This layer of oxide acts as an electrical insulator between the single-crystal silicon substrate and the first polycrystalline silicon layer (MMPoly0). A 0.8 $\mu \mathrm{m}$ thick layer of low-stress silicon nitride $\left(\mathrm{SiN}_{\mathrm{x}}\right)$ is deposited on top of the oxide layer. The nitride layer acts as an etch stop protecting the underlying oxide from wet etchants during processing. A $0.3 \mu \mathrm{m}$ thick layer of doped polycrystalline silicon ( $\mathrm{Si}$ ) known as: MMPoly0 is deposited on top of the nitride layer. MMPoly0 is not a structural layer, but it is usually pattemed and is used as a mechanical anchor, electrical ground, or electrical wiring layer. Following MMPoly0 deposition, the first sacrificial layer of $2 \mu \mathrm{m}$ oxide ('SacOxl) is deposited. Upon deposition of SacOx1, dimples are patterned and etched into the oxide. The dimples (primarily used for anti-stiction purposes) are formed from MMPolyl (the next polysilicon deposition). The dimple depth is approximately $1.5 \mu \mathrm{m}$. Figure $6 \mathrm{a}$ shows a crosssection of the wedge stepper motor following the dimple etch.

Following the dimple etches, the SacOxl is patterned and etched to form anchor sites through the depth of the oxide to the MMPoly0 layer. Polysilicon deposited over the SacOxl layer will be anchored or bonded to MIMPoly0 at the SacOxl cuts, and will also act as an electrical connection between MMPoly0 and MMPoly 1 . With the anchor sites defired, a $1 \mu \mathrm{m}$ thick layer of doped poly (MNIPolyl) is deposited. Figure 2 shows the comporients of the wedge stepper motor constructed out of MMPoly1.

Figure $6 \mathrm{~b}$ depicts the deposition and patterning of $0.5 \mu \mathrm{m}$ oxide ( $\mathrm{SacO} 2$ ) on top of MMPoly1. The SacOx2 layer provides a conformal coating both on top of MMPOPlyl and around the perimeter of the MMPolyl cuts.
Upon completion of the SacOx2 deposition, pattern, and etch, a $1.5 \mu \mathrm{m}$ thick layer of doped polysilicon, MMPoly2 is deposited. Following MMPoly2 deposition, an anisotropic reactive ion etch is performed to etch MMPoly2 and composite layers of MMPoly1 and MMPoly2 (laminated together to form a single layer approximately $2.5 \mu \mathrm{m}$ thick). Figure 2 shows the components of the wedge stepper motor constructed out of MMPoly2, and Figure 6c shows the cross-section of the wedge stepper motor after the MMPoly2 etch.

With the MMPoly2 etch complete, approximately $6 \mu \mathrm{m}$ of TEOS oxide ( $\mathrm{SacO} \times 3$ ) is deposited. Chemical-mechanical polishing (CMP) is used to planarize the oxide to a thickness of about $2 \mu \mathrm{m}$ above the highest point of MMPoly2. Following planarization, $\mathrm{SacO} \times 3$ is patterned and etched to provide dimples and anchors to the MMPoly2 layer. Figure 6d depicts the cross-section of the wedge stepper motor following $\mathrm{SacO} 33$ etch.

A $2 \mu \mathrm{m}$ thick layer of doped poly (MMPoly3) is deposited on the CMP planarized SacOx3. MMPoly 3 is patterned and etched to form the final structural layer. Figure 2 shows the components of the wedge stepper motor constructed out of MMPoly3, and Figure 6e shows MMPoly3 following deposition, anneal, pattern and etch.

Release and drying are the final fabrication steps. The wedge stepper motor is released by etching all the exposed oxides away with a 100:1 HF: $\mathrm{HCl}$ wet etch. Following the wet release etch, a drying process is employed using either simple air evaporation, supercritical $\mathrm{CO}_{2}$ drying, or $\mathrm{CO}_{2}$ freeze sublimation. The released wedge stepper motor is shown in Figure $6 \mathrm{f}$.

Figures 2 and 7 illustrate the independent use of all the mechanical layers available in the SUMMiT fabrication process.

\section{Device Operational Testing}

The device shown in Figure 2 was operationally tested to verify proper device function. This device was fabricated with no cover over the internal teeth so operation of the internal teeth and reciprocating shuttle could be visually observed. The speed of the reciprocating shuttle was increased to determine the operational limit and mode of any operation 
anomalies. The device demonstrated the ability to index at a speed of up to 205 gea: teeth per second. At higher speeds, the reciprocating shuttle tooth would bounce over the top of the internal teeth. This problem can be eliminated using an upper level cover (MMpoly3) over the internal teeth to constrain motion of the shuttle tooth during high-speed operation.

\section{Summary}

A wedge stepper motor was designed, fabricated and tested. This device can operate at speeds up to 205 indexes per second. The wedge stepper motor can very accurately hold the outer gear when the reciprocating shuttle is at the extreme of this motion. The device fabricated was $250 \mu \mathrm{m}$ in diameter, with 63 teeth and it could index the gear in increments of $2.85 \%$ step. The resolution of the angular indexing step size and the applied torque will increase with the outer gear diameter, which allows for a greater number of internal teeth. The concept of the wedge stepping motor can be applied to other configurations such as a linear rack. Additional variations of the wedge stepping motor have been designed and are being fabricated.

\section{Acknowledgements}

Sandia is a multiprogram aboratory operated by Sandia Corporation, a Lockheed Martin Company, for the United Stated Department of Energy under Contract DE-
AC04-94AL85000. The authors would like to thank the personnel of Sandia's Microelectronics Development Laboratory for the fabrication of the device described in this paper.

\section{References}

1. Multi-level polysilicon surfacemicromachining technology: applications and issues, J. J. Sniegowski, (Invited Paper) ASME 1996, International Mechanical Engineering Congress and Exposition, Proc. of the ASME Aerospace Division, November 17-22, 1996, Atlanta, GA, ADVol. 52, pp. 751-759.

2. Kinematics and Dynamics of Machines, $G$. H. Martin, McGraw-Hill, Inc., 1982.

3. J. J. Sniegowski, S. M. Miller, G. F. LaVigne, M.S. Rodgers and P. J. McWhorter, "Monolithic GearedMechanisms Driven by a Polysilicon Surface-micromachined On-Chip Electrostatic Microengine, " Solid-State Sensor and Actuator Workshop, Hilton Head Is., South Carolina, June 2-6, 1996, pp. 178182.

4. M. A. Polosky, E. J. Garcia, J. J. Allen, "Surface Micromachined Counter-Meshing Gears Discrimination Device", SPIE Smart Electronics and MEMS, San Diego, CA, Mar. 1998. 